\title{
Inkjet printed ECG electrodes for long term biosignal monitoring in personalized and ubiquitous healthcare
}

Link to publication record in Manchester Research Explorer

\section{Citation for published version (APA):}

Batchelor, J. C., \& Casson, A. J. (2015). Inkjet printed ECG electrodes for long term biosignal monitoring in personalized and ubiquitous healthcare. In host publication

\section{Published in:}

host publication

\section{Citing this paper}

Please note that where the full-text provided on Manchester Research Explorer is the Author Accepted Manuscript or Proof version this may differ from the final Published version. If citing, it is advised that you check and use the publisher's definitive version.

\section{General rights}

Copyright and moral rights for the publications made accessible in the Research Explorer are retained by the authors and/or other copyright owners and it is a condition of accessing publications that users recognise and abide by the legal requirements associated with these rights.

\section{Takedown policy}

If you believe that this document breaches copyright please refer to the University of Manchester's Takedown Procedures [http://man.ac.uk/04Y6Bo] or contact uml.scholarlycommunications@manchester.ac.uk providing relevant details, so we can investigate your claim.

\section{OPEN ACCESS}




\title{
Inkjet printed ECG electrodes for long term biosignal monitoring in personalized and ubiquitous healthcare
}

\author{
John C. Batchelor Senior Member, IEEE and Alexander J. Casson, Member, IEEE
}

\begin{abstract}
This paper investigates the performance of inkjet printed electrodes for electrocardiogram (ECG) monitoring in personalized and ubiquitous healthcare. As a rapid prototyping, additive manufacturing approach, inkjet printing can allow personalization of electrode sizes and shapes and can be used with a range of substrates to achieve good long term connections to the skin. We compare the performance of two types of inkjet electrodes printed using different substrates. Results demonstrate that both new electrodes can record ECG information, with comparable signal-to-noise ratios to conventional $\mathbf{A g} / \mathbf{A g C l}$ electrodes. The time-frequency decomposition of the collected signals is also explored.
\end{abstract}

\section{INTRODUCTION}

Wearable devices are starting to revolutionize personalized and preventative healthcare by allowing the easy, unobtrusive and long term monitoring of a range of body parameters. It is estimated that $90 \%$ of type 2 diabetes, $80 \%$ of heart diseases and $70 \%$ of strokes could be avoided with the use of suitable preventative techniques [1]. Activity trackers such as the fitbit [2] have been the most successful initial devices, enabled by the miniaturization and reduction in power consumption of electronics [3]. Further improvements in these factors, and the embedding of more complex signal processing, are now allowing smaller, more wearable, and more functional devices [4]. As a result there is a major opportunity to monitor more physiological parameters using wearable devices.

Heart monitoring is one such parameter. Monitoring via photophelsmograpgy is starting to appear in some devices, such as the Samsung Simband [5], but wearable electrocardiography (ECG) is much more challenging. Its better integration into wearable sensors is needed for studying heart function, cardiac arrhythmia, and oscillations during sleep, in the general population in a way not possible with traditional Holter monitors, but achieving this relies on having good wearable electrodes.

Traditional ECG uses wet $\mathrm{Ag} / \mathrm{AgCl}$ electrodes where a conductive gel is used to provide a resistive, low impedance, connection to the skin. These electrodes are well known for being easy to set up, but with limited lifetimes as the gel dries out. Capacitively coupled electrodes, which use an insulator gap instead of a conductive gel, have been researched since at least the 1960's [6]. They can be made as the back layer of a

This work was in part supported by the Engineering and Physical Sciences Research Council grant number EP/M009262/1.

J. C. Batchelor is with the School of Engineering and Digital Arts, University of Kent, UK. Email: j.c.batchelorekent.ac.uk.

A. J. Casson with the School of Electrical and Electronic Engineering, The University of Manchester, UK. Email: alex.casson@manchester.ac.uk.

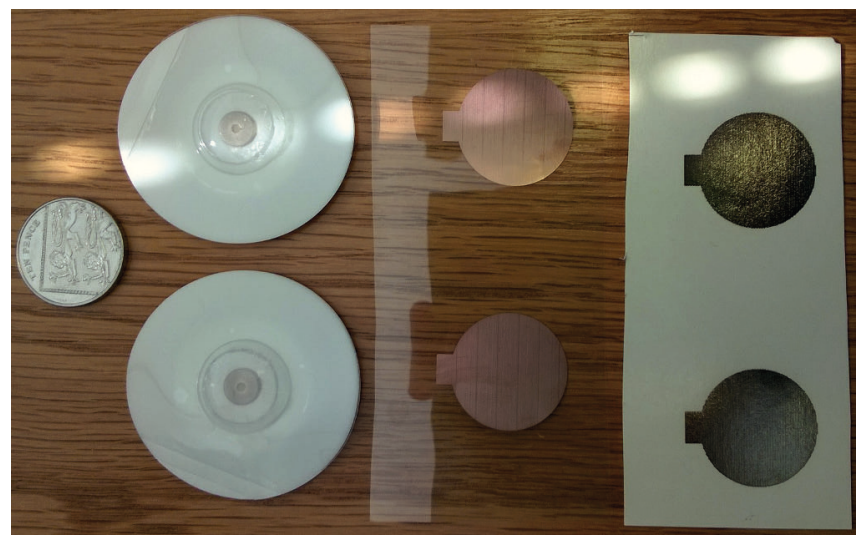

Fig. 1. ECG electrodes with a UK 10p piece for scale. (Left) Traditional $\mathrm{Ag} / \mathrm{AgCl} \mathrm{ECG}$ electrodes. (Middle) Copper printed capacitive electrodes. (Right) Silver printed electrodes on tattoo paper.

PCB [7] and so are easily personalisable, and the main focus recently has been on integrating capacitive electrodes into fabrics for smart clothing, (e.g. [8]). Nevertheless, these have yet to see widespread deployments. As an alternative, epidermal electrodes have been reported for tattoo-like monitoring of ECG [9]. However, these are based on Silicon processing, and are not directly suitable for low cost, personalizable electrodes.

This paper investigates inkjet printed electrodes as a hybrid solution between the two above. As a rapid prototyping, additive manufacturing approach, inkjet printing can allow personalization of electrode sizes and shapes and can also be used with a range of substrates to achieve tattoo-like connections to the skin. We compare the performance of two types of inkjet printed electrode, described in Section II. One is designed to be similar to current capacitive electrodes and one is designed to be similar to current tattoo-like electrodes. The performance comparison, Section III, demonstrates that both new electrodes can record ECG information, and the time-frequency decomposition for heart beat detection is explored.

\section{METHODS}

\section{A. Electrode manufacturing}

Three types of electrodes were compared in this study, shown in Fig. 1.

$\mathrm{Ag} / \mathrm{AgCl}$ : Traditional pre-gelled disposable $\mathrm{Ag} / \mathrm{AgCl} \mathrm{ECG}$ electrodes (Lessa, Spain) were used as the gold standard comparison case. These had a $7 \mathrm{~mm}$ diameter electrode at the center of a $50 \mathrm{~mm}$ diameter adhesive patch.

Capacitive: Inkjet printed capacitive ECG electrodes were made using a commercially available conductive inkjet tech- 
nology process [10], commonly used for the printing of antennas. The process is based upon using a catalytic ink on a flexible, transparent, plastic substrate and then using a plating solution to deposit copper on top of the catalysed areas [11]. It is an additive manufacturing approach with the advantages of rapid prototyping and little waste. Further, the plastic substrate is very flexible and conformal to the shape of the body, and the copper ink is highly conductive and can be directly soldered to. The disadvantage is that there is no inherent adhesive included, making it more difficult to maintain a fixed gap between the copper electrode and the body. Our printed electrodes were designed as a $25 \mathrm{~mm}$ circle with a $7 \mathrm{~mm}$ tab for electrode connection. ECG $1.5 \mathrm{~mm}$ touchproof connectors were attached to this tab using a conventional soldering process.

Tattoo: Tattoo style electrodes were inkjet printed using an in-house process with three layers of electrically conductive silver nano-particle paint, using similar procedures to [12] previously used for printing antennas [13]. The substrate in this case was temporary transfer tattoo inkjet paper with an adhesive film laid over it [14]. After printing this film provides a strong attachment to the skin and the backing paper of the temporary tattoo transfer material can be removed by wetting. This leaves behind the printed metal electrode on a very thin, approximately $10 \mu \mathrm{m}$, layer of plastic. This is an alternative an additive manufacturing approach. Compared to the capacitive electrodes the tattoos have the advantages of built-in adhesion and a thiner separation layer from the human body ${ }^{1}$. This is at the cost of the manufacturing process being less readily accessible.

The tattoo electrodes were printed using exactly the same design as for the capacitive electrodes. In this case the ECG touchproof connectors were attached using conductive silver paint with a layer of surgical tape placed over the connection point to strengthen the contact.

\section{B. ECG recordings}

To assess and compare the performance of the electrodes two electrode ECG traces were recorded using a CamNtech Actiwave (Cambridge, UK) ECG unit. The three types of electrodes were connected in turn for two hour recording periods. During this time the subject was stationary, using a computer for standard office work. This was followed by a test in which the subject walked around, allowing the investigation of motion artifacts in the collected traces. Electrodes were placed on the left and right sternum, equally spaced and approximately $3 \mathrm{~cm}$ from the mid-line. A third electrode, for impedance measurements, was placed on the mid-line, vertically in-line with the other two electrodes.

Both the $\mathrm{Ag} / \mathrm{AgCl}$ and tattoo electrodes are intrinsically adhesive and the electrodes were held in place using only this. The capacitive electrodes were covered with a microporous surgical tape which was used for the adhesion. When applying the capacitive and tattoo electrodes care was taken

\footnotetext{
${ }^{1}$ Note that the tattoo electrodes are capacitively coupled to the skin, like our copper ones, and so they are capacitive electrodes, but we termed them tattoo here in order to differentiate our two electrode types.
}

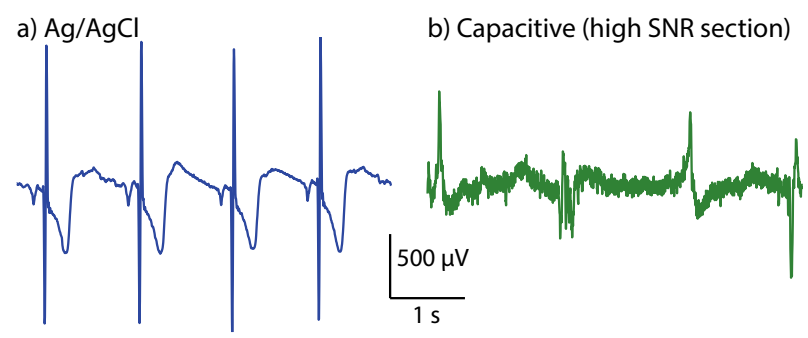

c) Capacitive (low SNR section)

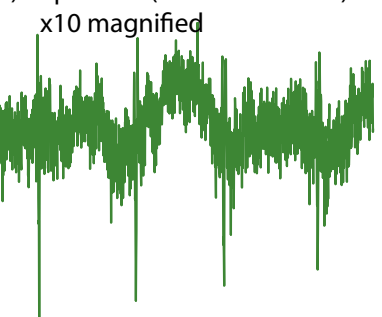

d) Tattoo

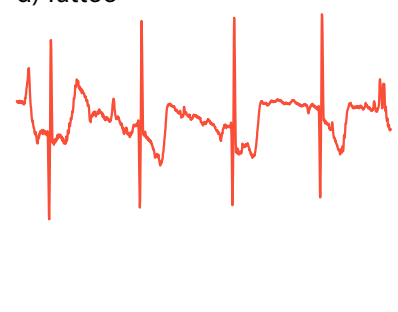

Fig. 2. ECG signals recorded from the three electrode types. Signals using $\mathrm{Ag} / \mathrm{AgCl}$ (a) and tattoo (d) electrodes are particularly clear. Heart beat times are clearly observable with the capacitive electrodes (b) and (c), although the Signal-to-Noise Ratio is substantially variable over time.

to ensure no air gaps were present underneath the electrode at the time of application.

All recordings used a 10 bit resolution, $1024 \mathrm{~Hz}$ sampling rate, downsampled to $256 \mathrm{~Hz}$ prior to analysis, and a $0.3 \mathrm{~Hz}$ high pass filter. The institutional review board in Manchester have approved Dr Casson to perform ECG measurements.

\section{PERFORMANCE ANALYSIS}

\section{A. Signal quality}

Example signals from the three electrode types are shown in Fig. 2. For the $\mathrm{Ag} / \mathrm{AgCl}$ electrodes (Fig. 2a) a clear R peak and heart beat are seen. This is also the case for the tattoo electrodes (Fig. 2d) although the full QRS complex is less clear with significant variations being present in the baseline between each beat. This effect is also present, and worse, for the capacitive electrodes (Fig. $2 b$ and c). With these electrodes R peaks are still clearly seen throughout the recording, but much powerline interference is present, substantially reducing the Signal-to-Noise Ratio (SNR). Moreover, the R peak amplitude is highly variable throughout the two hour recording, and as a result two separate sections of the trace are shown in Fig. 2 with different levels of magnification. This amplitude varied over time as the subject moved position. Both are non-ideal effects and are likely due to the connection using adhesive tape making it difficult to maintain a constant capacitive gap between the skin and the electrode as the subject moved, rather than due to the electrode itself.

For signal processing of the collected signals we are particularly interested in time-frequency approaches based upon the wavelet transform as we have previously demonstrated, Fig. 3, a custom microchip implementation using only $1.3 \mathrm{nW}$ of power [15], [16]. R peak locations have thus been extracted in the both the time domain and timefrequency domain using the MATLAB findpeaks routine and the constraint that each detection must be separated by 


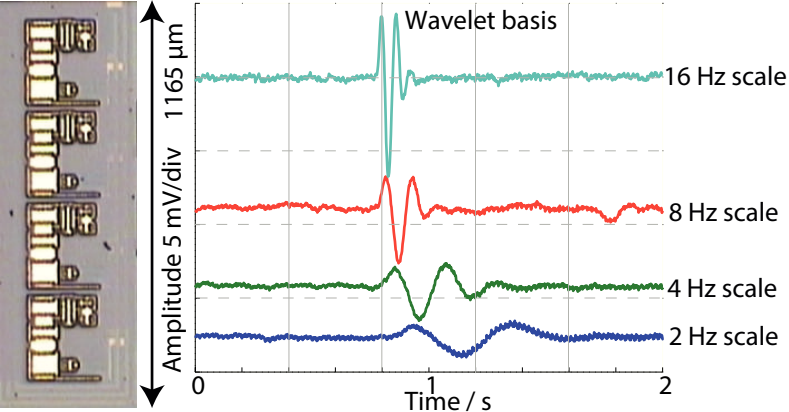

Fig. 3. Microchip implementation of a Mexican hat-like wavelet transform for real-time ECG analysis using $1.3 \mathrm{nW}$ of power [16]

TABLE I

SignAL-TO-Noise Ratio BETWEen R PEAK AND BACKGROUND.

\begin{tabular}{ccccc}
\hline & \multicolumn{4}{c}{ Signal-to-Noise Ratio / dB } \\
& Mean & Max & Min & Standard deviation \\
\hline & & \multicolumn{4}{c}{ Ag/AgCl } \\
Time domain & 19.9 & 27.4 & 06.7 & 0.9 \\
CWT domain & 36.2 & 46.0 & 32.6 & 0.7 \\
\hline \multirow{3}{*}{ Time domain } & 12.7 & 33.9 & -25.4 & 5.6 \\
CWT domain & 12.9 & 29.8 & -05.4 & 3.8 \\
\hline \multirow{3}{*}{ Time domain } & 19.3 & 32.4 & 03.9 & 3.9 \\
CWT domain & 26.2 & 36.8 & 03.0 & 5.3 \\
\hline
\end{tabular}

at least $0.39 \mathrm{~s}$. The time-frequency approach finds peaks in the Continuous Wavelet Transform (CWT) power signal, derived as the squared wavelet coefficients calculated using a scale 10 Mexican hat mother wavelet. In both the time and time-frequency domains the amplitude of the detected $\mathrm{R}$ peak is compared to the root-mean-squared amplitude of the baseline signal between each peak. To remove components of the R peak itself the baseline is selected to start from $0.39 \mathrm{~s}$ after the current $\mathrm{R}$ peak until $0.25 \mathrm{~s}$ before the next $\mathrm{R}$ peak. Comparing these two values gives an SNR which is evaluated over the first 45 minutes of the recording (before any substantial movement artifacts are present) to give a measure of the signal quality over time. The results are summarised in Table I.

For all three electrode types high mean SNRs are achieved indicating good quality signals and that it is possible to detect $\mathrm{R}$ peaks for heart rate and heart rate variability analyses. Similarly, in all cases an improvement in SNR is achieved by performing the analysis in the time-frequency domain. The Mexican hat mother wavelet, and consequently the processing provided by the circuit in Fig. 3, is particularly suited for this. The $\mathrm{Ag} / \mathrm{AgCl}$ electrodes have the highest SNRs and the lowest standard deviations demonstrating that they get the best performance, as would be expected from the clinical gold standard. Nevertheless, both the capacitive and tattoo electrodes have feasible SNRs, and indeed the time domain SNR of the tattoo electrodes is only marginally below that of the $\mathrm{Ag} / \mathrm{AgCl}$ ones. Both the capacitive and tattoo electrodes have a much wider variance in performance over time compared to the $\mathrm{Ag} / \mathrm{AgCl}$ and as a result for both electrode types the maximum SNR in the time domain is
TABLE II

RESIDUAL BASELINE WANDER OVER TWO HOURS

\begin{tabular}{ccccc}
\hline \multirow{2}{*}{ Electrode type } & \multicolumn{4}{c}{ Baseline wander $/ \mu \mathrm{V}$} \\
& Mean & Max & Min & Standard deviation \\
\hline $\mathrm{Ag} / \mathrm{AgCl}$ & 431 & 800 & -557 & 79 \\
Capacitive & -007 & 376 & -1425 & 83 \\
Tattoo & 048 & 1205 & -719 & 96 \\
\hline
\end{tabular}

higher than the maximum with the $\mathrm{Ag} / \mathrm{AgCl}$. In terms of signal quality there is clear potential for printed electrodes to provide good quality recordings. If it can be verified that the high SNR periods are not rare events, or outliers, higher SNRs than when using current $\mathrm{Ag} / \mathrm{AgCl}$ electrodes could even be possible. (See also Section III-D.)

\section{B. Impedance}

Electro-physiological standard three electrode impedance measurements were taken using a SIGGI II meter (EasyCap, Germany). For $\mathrm{Ag} / \mathrm{AgCl}$ electrodes the impedance at $30 \mathrm{~Hz}$ was $96 \mathrm{k} \Omega$ at first attachment, falling to $67 \mathrm{k} \Omega$ after two hours of use. In contrast both the capacitive and tattoo electrodes had impedances above $200 \mathrm{k} \Omega$, beyond our measurement capability, at the start and end of the recording. Electrode impedance is related to the amount of powerline $(50 / 60 \mathrm{~Hz})$ interference [17], and this is likely to be the single largest factor in the mean SNR reduction (Table I) during a two electrode ECG.

\section{Baseline wander}

The residual baseline wander was estimated by performing a six level Discrete Wavelet Transform (DWT) on the full 2 hour recorded traces. The level six approximation coefficients, which show the residual signal components in the $<2 \mathrm{~Hz}$ range, were median filtered using a $10 \mathrm{~s}$ moving window to remove any residual peaks due to heart beats and then filtered using a Kaiser window FIR to establish the low frequency changes in the baseline of the recorded signal. The residual baseline wanders are summarized in Table II.

The $\mathrm{Ag} / \mathrm{AgCl}$ electrodes have the worst mean performance, possibly indicating that some transient components of the heart beat are still present in the level six approximation which would increase the average found. Our two electrodes, which use capacitive rather than resistive coupling, have much lower levels of mean residual wander. Inevitably this comes at the cost of having a higher variance with the tattoo electrodes being the least constant. In all cases however the standard deviations are comparatively close and the printed electrodes do not perform substantially worse than conventionally manufactured ones.

\section{Movement artifacts}

Example signal traces from the walking test period are shown in Fig. 4 (top) when using the $\mathrm{Ag} / \mathrm{AgCl}$ and tattoo electrodes. With the capacitive electrodes our amplifier (with $8 \mathrm{mV}$ peak-to-peak range) saturated on each footfall making it impossible to apply further signal processing for analysis. 


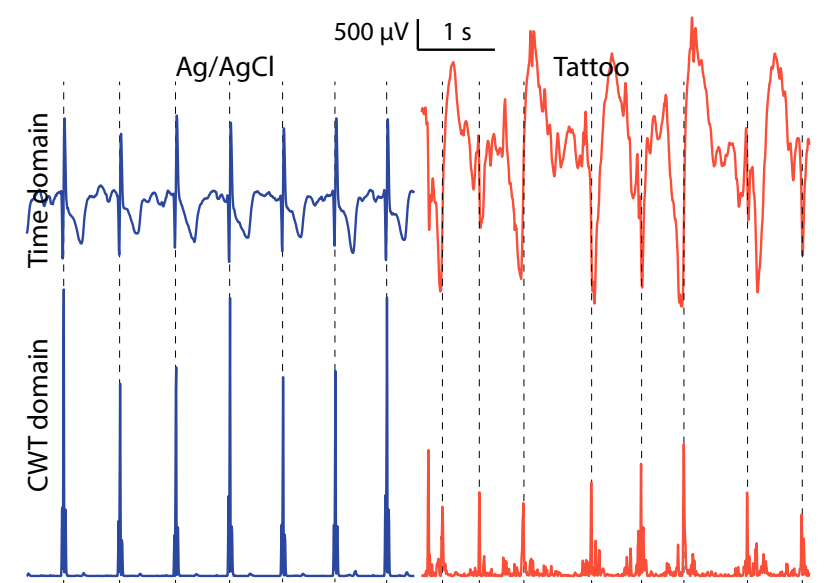

Fig. 4. ECG signals recorded during walking using $\mathrm{Ag} / \mathrm{AgCl}$ (left) and tattoo (right) electrodes. Signals are shown in both the time domain and the time-frequency domain.

With the $\mathrm{Ag} / \mathrm{AgCl}$ electrodes the $\mathrm{R}$ peaks remain clear throughout the walking period in both the time and timefrequency domains. Movement artifacts manifest as larger changes in the baseline between beats, but there is no substantial SNR reduction. With the tattoo electrodes (Fig. 4 right) substantial motion artifacts are seen, and these manifest in the CWT domain (squared wavelet coefficients using the scale 10 Mexican hat mother wavelet). As a result we have not yet achieved reliable heart rate detection using these sections of data. Nevertheless, some R peaks can be seen in the tattoo electrode time domain despite the presence of movement artifacts. Once motion artifact correlated signals (accelerometer or impedance) have been collected simultaneously we anticipate that the heart beat locations will be recoverable using adaptive filter techniques already established for ECG signals [18].

\section{E. Comfort and longevity}

We did not perform a formal survey of comfort or time to self-detachment of the electrodes. For the $\mathrm{Ag} / \mathrm{AgCl}$ these will be governed by the gel drying out, and for the capacitive electrodes by the adhesive used. Nevertheless, anecdotally we highlight the exceptional longevity of the tattoo approach. One subject kept the electrodes on after the experiment, and they were still attached five days after later, having been through showers on intervening days. Further, on day 5 the subject did a 20 mile run and the electrodes were still attached at the end. While formal investigation is required, there is substantial potential for printed electrodes to enable chronic ECG recordings over time spans not currently possible.

\section{CONCLUSIONS}

This paper has investigated the performance of two types of printed ECG electrode compared to $\mathrm{Ag} / \mathrm{AgCl}$ ones. Both new electrodes were able to record ECG signals which were suitable for time-frequency analysis using the wavelet transform, potentially provided by ultra low power CWT circuits operating in the nano-Watt and sub-nano-Watt range. High Signal-to-Noise ratios were obtained for the two types of printed electrodes, but at the expense of the SNR being much more variable and susceptible to motion interference. The tattoo printed electrodes were particularly promising for long term ECG recording. Both types of printed electrode showed substantial promise for the rapid and low cost manufacture of personalized electrodes for future wearable ECG.

\section{ACKNOWLEDGMENTS}

The authors would like to acknowledge and thank CIT Cambridge for printing the capacitive electrodes, and Rachel Saunders of the School of Materials Science at the University of Manchester for printing the tattoo electrodes.

\section{REFERENCES}

[1] A. Honka, K. Kaipainen, H. Hietala, and N. Saranummi, "Rethinking health: ICT-enabled services to empower people to manage their health," IEEE Rev. Biomed. Eng., vol. 4, no. 1, pp. 119-139, 2011.

[2] fitbit. (2014) Home page. [Online]. Available: http://www.fitbit.com/

[3] S. A. Imtiaz, A. J. Casson, and E. Rodriguez-Villegas, "Compression in wearable sensor nodes: Impacts of node topology," IEEE Trans. Biomed. Eng., vol. 61, no. 4, pp. 1080-1090, 2014.

[4] G. Chen, E. Rodriguez-Villegas, and A. J. Casson, "Wearable algorithms: An overview of a truly multi-disciplinary problem," in Wearable sensors, E. Sazonov and M. R. Neuman, Eds. Amsterdam: Elsevier, 2014, pp. 353-382.

[5] Simband. (2015) Home page. [Online]. Available: http://www. voiceofthebody.io/

[6] A. Lopez and P. C. Richardson, "Capacitive electrocardiographic and bioelectric electrodes," IEEE Trans. Biomed. Eng., vol. 16, no. 1, p. 99 , 1969.

[7] Y. M. Chi, Y.-T. Wang, Y. Wang, C. Maier, T.-P. Jung, and G. Cauwenberghs, "Dry and noncontact EEG sensors for mobile brain-computer interfaces," IEEE Trans. Neural Syst. Rehab. Eng., vol. 20, no. 2, pp. 228-235, 2012.

[8] L. Yan, J. Yoo, B. Kim, and H.-J. Yoo, "A $0.5-\mu \mathrm{V}_{r m s} 12-\mu \mathrm{W}$ wirelessly powered patch-type healthcare sensor for wearable body sensor network," IEEE Trans. Syst. Sci. Cybernetics, vol. 45, no. 11, pp. 2356-2365, 2010.

[9] D.-H. Kim, N. Lu, R. Ma, Y.-S. Kim, R.-H. Kim, S. Wang, J. Wu, S. M. Won, H. Tao, A. Islam, K. J. Yu, T.-i. Kim, R. Chowdhury, M. Ying, L. Xu, M. Li, H.-J. Chung, H. Keum, M. McCormick, P. Liu, Y.-W. Zhang, F. G. Omenetto, Y. Huang, T. Coleman, and J. A. Rogers, "Epidermal electronics," Science, vol. 333, no. 6044, pp. 838-843, 2011.

[10] InkJetFlex. (2015) Home page. [Online]. Available: http://inkjetflex. $\mathrm{com} /$

[11] CIT technology. (2015) Conductive inkjet printing. [Online]. Available: http://cittechnology.com/flexible-circuits/ conductive-inkjet-printing.html

[12] V. Sanchez-Romaguera, M. A. Ziai, D. Oyeka, S. Barbosa, J. S. R. Wheeler, J. C. Batchelor, E. A. Parker, and S. G. Yeates, "Towards inkjet-printed low cost passive UHF RFID skin mounted tattoo paper tags based on silver nanoparticle inks," J. Mater. Chem. C, vol. 1 , no. 39, pp. 6395-6402, 2013.

[13] M. A. Ziai and J. C. Batchelor, "Temporary on-skin passive UHF RFID transfer tag," IEEE Trans. Antennas Propagat., vol. 59, no. 10, pp. 3565-3571, 2011.

[14] Crafty computer paper. (2015) Inkjet tattoo paper. [Online]. Available: http://www.craftycomputerpaper.co.uk/category/ Temporary-Tattoo-Paper

[15] A. J. Casson and E. Rodriguez-Villegas, "A $60 \mathrm{pW} g_{m} C$ Continuous Wavelet Transform circuit for portable EEG systems," IEEE J. SolidState Circuits, vol. 46, no. 6, pp. 1406-1415, 2011.

[16] _- "Nanowatt multi-scale continuous wavelet transform chip," Electron. Lett., vol. 50, no. 3, pp. 153-154, 2014.

[17] T. C. Ferree, P. Luu, G. S. Russell, and D. M. Tucker, "Scalp electrode impedance, infection risk, and EEG data quality," Clin. Neurophysiol., vol. 112, no. 3, pp. 536-544, 2001.

[18] P. S. Hamilton, M. G. Curley, R. M. Aimi, and C. Sae-Hau, "Comparison of methods for adaptive removal of motion artifact," in CinC Memphis, September 2000. 